\title{
Virtudes y debilidades en la mirada del Capital Humano en las Instituciones de Educación Superior
}

\author{
Strengths and weaknesses of the Human Capital approach in Higher \\ Education Institutions
}

\begin{abstract}
Jorge Maluenda Albornoz ${ }^{1}$
RESUMEN

El presente ensayo analiza el Enfoque del Capital Humano en la educación superior contraponiendo argumentos que defienden su valor y advierten de sus problemas. El objetivo que persigue es analizar las virtudes y debilidades más relevantes del Enfoque del Capital Humano en el contexto de la educación superior latinoamericana. El análisis muestra cómo es posible observar grandes contribuciones de este enfoque en cuanto a la gestión estratégica y eficiente de la enseñanza y administración de la educación superior, la valorización de los intangibles y la consideración del entorno. También, muestra como principales problemas una tendencia a la reducción de los propósitos humanos, el utilitarismo y el alineamiento potencial de los estudiantes y futuros profesionales. Se concluye la existencia de contribuciones relevantes de este enfoque a la gestión educativa, especialmente necesarios, considerando la escasez de recursos y el ingreso masivo de los estudiantes durante los últimos 30 años en Latinoamérica. Estos pueden ser aprovechados de mejor manera cuando no se pierden de vista los propósitos originales y más importantes de la educación superior.
\end{abstract}

Palabras clave: Educación superior, capital humano, utilitarismo, gestión educativa, accountability.

\begin{abstract}
This essay analyzes the human capital approach to higher education, contrasting arguments that defend its value and warn of its problems. Its objective is to analyze the most relevant strengths and weaknesses of the human capital approach in the context of Latin American higher education. The analysis shows how it is possible to observe great contributions of this approach in terms of the strategic and efficient management of the teaching and administration of higher education, the valuation of intangibles and the consideration of the environment. Also, it shows as main problems a tendency to reduce human purposes, utilitarianism and the potential alignment of students and future professionals. The existence of relevant contributions of this approach to educational management is concluded, especially necessary, considering the scarcity of resources and the massive admission of students during the last 30 years in Latin America. These can be put to best use when the original and more important purposes of higher education are not lost sight of.
\end{abstract}

Key Words: Higher education, human capital, utilitarianism, educational management, accountability.

\section{Introducción}

\footnotetext{
${ }^{1}$ Universidad San Sebastián. Concepción, Chile. Magíster en Política y Gobierno. Candidato a doctor en Psicología. Académico de la Facultad de Psicología. Correo electrónico: jmaluendaa@ docente.uss.cl ORCID: https://orcid.org/0000-0001-8148-4948

DOI: $10.15517 /$ WL.V1711.49499

Recepción: 9/8/2021 Aceptación: 30/10/2021
} 
La mirada del Capital Humano es un enfoque que, actualmente, se encuentra bastante instalado, no solo en la institucionalidad y las prácticas concretas, sino que, además, en la cultura de las Instituciones de Educación Superior (IES) (Acevedo, 2018).

Ha obtenido un lugar relevante y ha adquirido fuerza debido a sus virtudes para focalizar la dirección de las actividades institucionales y los esfuerzos, de forma eficiente, con foco en la obtención de resultados mesurables.

Tanto es así que en la llamada sociedad del conocimiento "las IES consideran como prioridad la calidad del know how de las personas y sus demás competencias y habilidades; es decir, el capital humano (...) Esto adquiere mayor relevancia en la universidad, donde uno de sus fines es la producción y generación de nuevo conocimiento en beneficio de la sociedad"1. (Cuentas, 2018, p.58).

Como en todo paradigma, existen ventajas y desventajas tanto en su propuesta como en sus métodos. Luces y sombras que toda organización, líder, gestor, académico y profesional debiese tener en cuenta de modo que permita aprovechar sus virtudes y resguardarse de sus problemas.

\section{Discusión}

Una de las virtudes indiscutibles es la capacidad de este enfoque para organizar y administrar los esfuerzos organizacionales con dirección al logro de resultados. El advenimiento de la gestión por competencias en su versión adaptada a la educación - Enfoque Basado en Competencias - (Villaroel y Bruna, 2014) y la mirada de los Resultados de Aprendizaje (Gaebel y Zhang, 2018) son muestras de la importancia adquirida del establecimiento de rutas organizadas y eficientes de gestión educativa, como esfuerzo por orientar a las IES a la obtención de mejores resultados.

En Latinoamérica, esta ventaja adquiere mayor relevancia cuando las carencias económicas y la enorme heterogeneidad de necesidades realzan la importancia de una gestión eficiente. Evidencia de ello es la fuerza adquirida desde 2004 del denominado Proyecto Tuning en Latinoamérica (Beneitone et al., 2007), el realce adquirido por agencias nacionales e internacionales de acreditación en los distintos países latinoamericanos (Gutiérrez, 2005) e incluso, en el caso de algunos países, legislaciones que obligan a las IES a avanzar en dicha 
dirección (Ej. Decreto 1330 de 2019 sobre Resultados del Aprendizaje en Colombia; Ley 20129 de 2006 sobre el Sistema de Aseguramiento de la Calidad en Educación Superior Chile).

Queda aparte el necesario análisis para evaluar si a 17 años de su origen en esta región del planeta el enfoque formativo ha alcanzado los resultados anhelados, aunque, sin dudas, ha generado un potente impacto en el paradigma educativo, en las preocupaciones de las IES y de los académicos, en las prácticas y en todo un nuevo nicho laboral (y de negocios) asociados a las acreditaciones (Maluenda, 2021).

Una segunda virtud es que, al ser un enfoque centrado en los resultados, ha obligado al desarrollo de una gestión que observe y considere como aspecto fundamental la incorporación de la mirada externa y de las necesidades reales de la industria, las comunidades y el entorno tanto local como global (Cuentas, 2018).

Una crítica a la academia vinculada con esta segunda virtud, clásica ya a estas alturas, ha sido el excesivo academicismo de las IES (Caballero, 2014) que ha limitado su capacidad para adaptarse a los nuevos tiempos, así como también le ha distanciado de las necesidades inmediatas y reales. En este problema se sustenta una de las piedras angulares del proceso de Lisboa y el Tratado de Bolonia en Europa puesto que generó una desconexión y desafición evidenciable, que llevó a titular cohortes de profesionales con escasas capacidades para enfrentar los requerimientos reales de la época (Maluenda, 2021).

La mirada del Capital Humano exige observar el mundo para adaptar tanto los propósitos educativos como las actividades organizativas que pretendan alcanzarlos frente a los requerimientos del entorno. Adquieren valor herramientas de la administración organizacional para comprender y analizar las tendencias que lleven a ajustar las propias actividades para que sean significativas frente a los requerimientos de la industria, la comunidad, la tecnología, el ambiente, etc.

Desde este punto de vista, el enfoque del capital humano en educación ha promovido un diálogo más fluido y permanente entre los stakholders y las IES ha favorecido el perfeccionamiento permanente de las estrategias de enseñanza, la vinculación con prácticas y pasantías en organizaciones de la sociedad civil y también, una reevaluación de los propósitos formativos. Con todo ello, se genera una educación más actualizada y contextualizada a las necesidades y demandas propias de la sociedad. 
Una tercera ventaja observable es el realce y la valoración de los "intangibles" como aspectos fundamentales en la formación. El insight por parte de las IES detrás de esta ventaja tiene relación con la observación de que, profesionales técnicamente bien calificados no obtenían los resultados esperados debido a una carencia en la formación transversal o genérica a pesar de su sólida formación técnica (Maluenda, 2021).

La inhabilidad para comunicarse efectivamente, negociar, resolver conflictos, lidiar con la incertidumbre y la frustración, organizarse y trabajar en equipos efectivos produjo efectos tanto en el desempeño como desajustes en la organización generando conflicto y malestar entre colegas y empleadores. Más aún, los nuevos tiempos trajeron consigo nuevas necesidades como las capacidades asociadas a la autogestión, la autoformación permanente, la capacidad para la iniciativa innovadora y, por supuesto, el dominio de las herramientas tecnológicas necesarias para el trabajo. Estas brechas fueron alimentadas por el cambio en los perfiles de ingreso de los estudiantes gracias al acceso masivo a la Educación Superior iniciado a comienzos de los 90's en Latinoamérica y la lenta respuesta frente a este cambio demográfico de parte de los gobiernos y las IES (Brunner y Miranda, 2016).

Las desventajas que trae consigo la formación desde el Enfoque del Capital Humano son menos visibles, aunque convivan con nuestra práctica diaria, razón por la que se hace especialmente relevante tornarlas visibles y advertir de sus problemas. Estas subsisten en la visión paradigmática que conlleva efectos prácticos como se plantea a continuación.

En primer lugar, el Enfoque del Capital Humano en educación trae consigo una visión específica sobre el ser humano, sus propósitos y razones. Desde esta perspectiva el ser humano es concebido como un factor de producción que puede - y debe - ser aprovechado para bien de los objetivos organizacionales (Dădârlat y Dumitraşcu, 2015; Sandoval y Hernández, 2018). Desde el Management se hace necesario invertir y desarrollar este capital humano para generar mayor eficiencia y conducir a mejores resultados organizacionales (Acevedo, 2018).

Esta visión es transferida al plano formativo donde las IES deben organizar la formación para desarrollar adecuadamente el potencial laboral de los estudiantes, de modo que, una vez incorporados al mundo del trabajo, estos cuenten con las capacidades que la industria requiere. Aún más, esta visión permea el perfeccionamiento continuo y la necesidad 
permanente de un vínculo entre la academia y la industria, para actualizar y desarrollar las capacidades de las personas con orientación al trabajo.

De hecho, las IES son un pilar fundamental del modelo puesto que son estas las que proveen el capital humano a la industria, quienes además perfeccionan y actualizan sus capacidades, basadas en el desarrollo permanente de conocimiento de vanguardia (Cuentas, 2018).

Las personas entonces se reducen a “(...) un recurso vital de todas las organizaciones, sin importar el campo de su actividad, un recurso que les asegura la supervivencia, el desarrollo y el éxito en la competitividad" (Dădârlat y Dumitraşcu, 2015, p.85), lo que genera un reduccionismo de los propósitos humanos, circunscritos al trabajo y la producción, excluyendo de la ecuación aspectos como el desarrollo personal, el bienestar y el ocio.

En segundo lugar, el Enfoque del Capital Humano viene de la mano con una visión sobre el aprendizaje y los saberes como "mercancías" que se vincula con lo que se ha denominado anteriormente el "capitalismo de los bienes inmateriales" (Castells, 1999). La asociación entre la economía y el conocimiento es una relación construida desde la revolución industrial que, durante el siglo $\mathrm{XX}$, ha generado el relato y las lógicas necesarias para tratar el conocimiento, las relaciones y los afectos como bienes materiales (Maluenda, 2018).

Un proceso de capitalización hipotético busca determinar qué atributos permiten la legítima apreciación del conocimiento y el potencial humano. Esta sería posible gracias a la transferencia de la lógica de la escasez sobre lo inmaterial: a) se establece lo inmaterial como bien rival (solo puede pertenecer a un dueño); b) se entiende lo inmaterial como bien de uso exclusivo (solo el dueño puede decidir quién lo usa). Gracias a la legislación y las regulaciones es posible aplicar estos principios al aprendizaje y los saberes (Maluenda, 2018).

Este peligroso utilitarismo puede dotar de una relato alienante, vacío y extremadamente funcional a aspectos clave de la vida en humana como son el desarrollo personal, el potencial humano y el valor de las relaciones. Al mismo tiempo, transforma intangibles como la confianza, las buenas relaciones interpersonales - y las habilidades requeridas para establecerlas - el poder del liderazgo, las convicciones, el aprendizaje e incluso el bienestar en aspectos cuyo propósito principal (y muchas veces único) es la producción. 
Vinculado a estos relatos aparecen ideas como el potencial indefinido - e inagotable - de la persona, el mérito como sistema de progreso, el nivel socioeconómico como medida de desarrollo personal, e incluso, como propósito vital.

En la contracara a los beneficios de la gestión educativa antes planteados, una visión extrema puede generar una deformación en el accountability que reduce los procesos de administración en educación a la mera medición de indicadores - muchas veces carentes de sentido y utilidad - como propósitos en sí mismos. De este modo se generan procesos vacíos porque las acciones de medición no contribuyen al proceso de gestión y menos a la formación de personas pero, además, porque quitan del escenario el valor del proceso y los propósitos más humanistas de la formación educativa.

La UNESCO ya en el año 2015 alertaba sobre este predicamento invitando a las IES a considerar los aportes del Enfoque del Capital Humano, pero también a redirigir los esfuerzos de su trabajo de gestión y formación hacia "una concepción más humanista de la educación que ponga su foco de atención en el respeto a la vida, la dignidad humana y el bienestar de las personas" (UNESCO, 2015).

\section{Conclusiones}

Teniendo en cuenta los diferentes cuestionamientos antes planteados es posible arribar a algunas conclusiones y proponer la discusión sobre las ventajas de la mirada del Capital Humano en el contexto de la formación de personas y de la orientación de las IES evitando sus complicaciones.

La implementación de estrategias basadas en la mirada del Capital Humano conlleva virtudes importantes cuando se utilizan sus herramientas de gestión y accontability para potenciar la gestión académica de forma eficiente. Además, permite un vínculo más fluido, cercano y permanente con el entorno y los stakeholders que les permita establecer alianzas y mantener una actualización permanente acorde a las necesidades formativas vigentes en la sociedad. Para aprovechar estas virtudes será importante mantener claridad en los propósitos educativos en orden de una formación sólida en lo técnico que conserve la mirada humanista de la educación. Además, será un desafío de las IES mantener una mirada propositiva sobre 
la Educación Superior, planteando desafíos, prefigurando el futuro y evitando responder solamente a las demandas del entorno.

Las IES se juegan la posibilidad de ejercer el rol social que corresponde a los centros de pensamiento y estudio en una sociedad, que es, ser actores transformadores de la sociedad con base en el estudio de las diversas disciplinas. Será parte de su reflexión definir cuál es el rol que cada institución pretende desempeñar en el contexto de la sociedad en la que se inscribe, su influencia en la discusión pública y su capacidad para "empujar" los límites de lo establecido.

Aspectos clave que deben ser parte de la discusión actual de las IES en la aplicación de la mirada del Capital Humano son: ¿Por qué y para qué implementar sistemas de gestión de la calidad educativa? ¿Qué entiende la institución por calidad? ¿Cómo construir procesos internos eficientes, pero siempre útiles a la mejora educativa? ¿Qué particularidades de nuestros contextos deben ser consideradas al momento de aplicar las estrategias? ¿Qué es importante conservar y qué aspectos son prescindibles?

Por otra parte, la revalorización de los intangibles que conlleva la mirada del Capital Humano permite que las IES hoy se enfoquen en diseñar estrategias formativas que les incluyan, validen y permitan formar profesionales realmente integrales. De este modo, las competencias genéricas/transversales dejan de ser "blandas" y se valoran de igual modo que las competencias disciplinares de cara a la formación profesional. Se valida la importancia de utilizar estrategias planificadas que muestren garantías de éxito y, además, surge la necesidad de habilitar a los docentes para que sean peritos en ellas empujando procesos de perfeccionamiento docente permanente.

Grandes desafíos surgen desde aquí. Por un lado, las IES deberán ser capaces de leer el dinamismo del entorno, el cambio permanente de los problemas (sociales, económicos, políticos y ambientales, etc.) y el progreso de la tecnología para poder responder de buena forma a las nuevas tendencias y requerimientos en cuanto a competencias. Por otro, deberán invertir en la instalación de procesos de perfeccionamiento docente y mejora continua asumiendo que su coste retribuye de manera sustantiva en distintos aspectos.

El gran desafío de las IES en la implementación de los aportes del Capital Humano en educación comprende la construcción de un relato que contribuya a una cultura que contemple sus virtudes - mirada estratégica, eficiencia, conexión con el entorno, 
adaptabilidad - protegiendo la educación del excesivo utilitarismo, funcionalismo y alienación de los individuos y los grupos humanos.

Una cultura que proteja el valor del aprendizaje como fin en sí mismo, la relevancia de las distintas formas del saber, el valor intrínseco de los procesos y el cuidado de un desarrollo integral de los seres humanos puede conducir al resguardo respecto de malas prácticas y excesos fundados en los preceptos del Capital Humano.

Algunos aspectos que las IES deberán cautelar son:

- La persecución de métricas e indicadores como fin último de la gestión educativa. Por lo tanto, jamás se debe olvidar la finalidad principal del proceso educativo.

- La orientación del proceso educativo como respuesta a un mero Market Pull dejando fuera la capacidad reflexiva y prefigurativa de la academia.

- La pérdida de visibilidad del valor que agregan fenómenos vinculados al proceso educativo como el disfrute del aprendizaje, el bienestar estudiantil y del profesorado, la generación de lazos de apoyo no instrumental, el valor no funcional del ocio y las artes, entre tantos otros.

- Tratar al saber, a los actores del proceso educativo y a los intangibles que este produce como mercancías. Por el contrario, promover el valor intrínseco que todos estos componentes conllevan y su contribución a la vida social por sobre la dimensión netamente económica.

Por último, para una futura reflexión será clave poner la mirada sobre los efectos culturales del enfoque en la vida laboral docente, sus alcances sobre la comprensión del rol académico en la actualidad y los márgenes que se deben dibujar para evitar deformaciones como las que se observan en cuanto a la actividad educativa propiamente dicha.

Un aspecto no tratado en este ensayo y de gran relevancia es cómo la mirada del Capital Humano puede afectar la gestión ocupacional de las y los docentes, y qué repercusiones puede ello tener en la orientación de sus actividades para lograr los propósitos de la educación. Preguntas que surgen inevitablemente desde este punto son: ¿Cómo afecta la mirada del Capital Humano las aspiraciones y el ejercicio de los docentes en la actualidad? ¿De qué modo influyen los nuevos modelos de gestión sobre la actividad laboral docente? Las contribuciones y los problemas de la aplicación de los preceptos del Capital Humano en Educación Superior denotan las "luces y sombras" de una propuesta filosófica y práctica para 
la orientación y gestión de procesos humanos. Como toda mirada, plantea desafíos de los cuales se debe ser consciente para aprovechar sus ventajas y evitar sus vicios en la implementación de nuestras prácticas habituales, con el objetivo de beneficiar al sistema educativo y los destinatarios de sus efectos, y así evitar desvirtuar los propósitos más primigenios y relevantes del quehacer educativo.

\section{Referencias}

Alexandra Dădârlat, Dănut Dumitraşcu, $<<$ The role and importance of human capital in contemporary organizations>>. Review of Management \& Economic Engineering. 14, 1 (2015): 78-86.

Andrés Acevedo, <<La teoría del capital humano, revalorización en educación: Análisis, evolución y críticas a sus postulados〉>. Revista Reflexiones y Saberes, 5, 8(2018): $58-72$.

Gloria Caballero, José López-Miguens, Jesús Lampón, <<La universidad y su implicación con la empleabilidad de sus graduados〉>. Revista Española de Investigaciones Sociológicas, 146(2014): 23-46

Jorge Maluenda-Albornoz, <<Valores y prácticas del sistema educacional chileno $>$. (manuscrito inédito, diciembre 2018). Archivo PDF.

Jorge Maluenda-Albornoz, <<Desafíos para la educación orientada al desarrollo de competencias 2.0>>. En Educación orientada al desarrollo de competencias. Guía para su implementación efectiva, editado por Jorge Maluenda-Albornoz [En prensa].

José Sandoval, Gustavo Hernández, <<Crítica a la teoría del capital humano, educación y desarrollo económico〉>. Revista Ensayos Pedagógicos 2, 13(2018):137-160.

José Brunner, Daniel Miranda, Educación superior en Iberoamérica: Informe 2016. Santiago: RIL Editores, 2016.

Manuel Castells, La sociedad red. Madrid: Alianza Editorial,1999.

Michael Gaebel, Thérèse Zhang, Learning and teaching in the European higher education area. Bruselas: European University Association, 2018.

Muriel Acevedo, <<La teoría del capital humano, revalorización de la educación: análisis, evolución y críticas de sus postulados>>. Revista Reflexiones y Saberes 5, 8(2018): $58-72$. 
Olga Gutiérrez, <<Educación y entrenamiento basados en el concepto de Competencia: implicaciones para la acreditación de los Programas de Psicología $>$. Revista Mexicana de Psicología, 22, 3(2005): 253-270.

Pablo Beneitone, César Esquetini, Julia González, Maida Marty, Gabriela Siufi, Robert Wagenaar, Reflexiones y perspectivas de la Educación Superior en América Latina. Bilbao: Publicaciones de la Universidad de Deusto, 2007.

Susan Cuentas, <<La gestión del capital humano en una unidad académica de educación superior. Análisis de la universidad>>. Educación, 27, 53(2018): 57-72., doi: 10.18800/educacion.201802.004

Thomas Davenport, Capital humano. Barcelona: Gestión 2000, 2006.

UNESCO, Replantear la educación ¿Hacia un bien común mundial? París: UNESCO, 2015.

Verónica Villarroel, Daniela Bruna, <<Reflexiones en torno a las competencias genéricas en educación superior: Un desafío pendiente〉>. Psicoperspectivas, 13, 1(2014): 23-34, doi: 10.5027/PSICOPERSPECTIVAS-VOL13SSUE1-FULLTEXT-335 